\title{
Registros de duas espécies de morcegos (Chiroptera: Molossidae) encontrados mortos em cercas de arame farpado no noroeste do estado de São Paulo, Brasil
}

\author{
Crasso Paulo Bosco Breviglieri \\ PPG em Biologia Animal, Ecologia e Comportamento Animal \\ Departamento de Zoologia e Botânica, IBILCE/UNESP \\ Rua Cristóvão Colombo, 2265, Jd. Nazareth, CEP 15054-000, São José do Rio Preto $\square$ SP, Brasil \\ crassopaulo@yahoo.com.br
}

Submetido em 05/04/2013

Aceito para publicação em 14/10/2013

\section{Resumo}

Em todo o mundo há descrição de casos nos quais morcegos são encontrados mortos devido a ações humanas. Os principais registros estão relacionados à influência de cercas de arame farpado, redes elétricas, pesticidas e turbinas eólicas. No Brasil, esses dados são pouco explorados e merecem mais atenção dos pesquisadores e agências governamentais. Esta nota tem o objetivo de descrever dois registros de morcegos (Molossus molossus e Molossus rufus) encontrados mortos em cercas de arame farpado, no noroeste do estado de São Paulo. Além disso, discute brevemente a possível relação entre esse tipo de acidente e a proximidade de cercas de arame farpado de áreas de forrageio ou de abrigos dessas espécies.

Palavras-chave: Arame farpado; Conservação; Morcego

\section{Abstract}

Records of two bat species (Chiroptera: Molossidae) found dead in barbed-wire fences in the northwestern São Paulo state, Brazil. Worldwide, there are descriptions of cases in which bats are found dead due to human actions. The main records are related to the influence of barbed-wire fences, electrical nets, pesticides, and wind turbines. In Brazil, these data are poorly explored and deserve more attention from researchers and government agencies. This note aims to describe two records of bats (Molossus molossus and Molossus rufus) found dead in barbed-wire fences, in the northwestern São Paulo state, Brazil. Furthermore, it briefly discusses the possible relation between this kind of accident and closeness of barbed-wire fences to foraging or shelter areas for these species.

Key words: Barbed-wire; Bat; Conservation 


\section{Introdução}

Ao redor do mundo, varias espécies de animais silvestres foram encontrados mortos em cercas de arame farpado, ou então foram resgatados com diversos ferimentos (MCNICHOLL 1988; VAN DER REE, 1999; NORQUAY et al., 2010; NAVO et al., 2011). Os grupos mais comumente registrados são aves (EMERSON, 1904; ANDERSON, 1977; VAN DER REE, 1999; ALLEN; RAMIREZ, 1990) e mamíferos (VAN DER REE, 1999). Casos onde morcegos são encontrados mortos em cercas de arame farpado, em redes elétricas, em rodovias ou após colidirem em edifícios, também são comumente relatados ao redor do mundo (DENYS, 1972; WISELY, 1978; DEDON et al., 1989; MACLEAN, 2006). No Brasil, registros desta natureza são escassos, podendo destacar apenas um relato referente a um indivíduo da espécie Vampyrum spectrum (Linnaeus, 1758) morto eletrocutado em Rondônia (DISCHER et al., 2009). Portanto o objetivo desta nota é descrever dois registros de morcegos pertencentes à família Molossidae, encontrados mortos em cercas de arame farpado, na região noroeste do estado de São Paulo, Brasil.

\section{Material e Métodos}

Estes registros foram obtidos de maneira fortuita durante campanhas de campo realizadas nas proximidades das cidades de São José do Rio Preto e Fernandópolis, ambas localizadas na região noroeste do estado de São Paulo. Após encontrar os morcegos, busquei identificar se existiam possíveis abrigos em um raio de $100 \mathrm{~m}$ do ponto onde estavam os animais. Também foram anotadas as datas dos registros e os exemplares foram coletados para confirmar a identificação em laboratório, devido ao seu presente estado de putrefação.

\section{Resultados}

O primeiro registro refere-se a uma fêmea da espécie Molossus molossus (Pallas, 1766) medindo 37,2 $\mathrm{mm}$ de antebraço e pesando 7,5 $\mathrm{g}$, encontrada morta no dia 23 de julho de 2010, na zona rural da cidade de São José do Rio Preto, na coordenada 2051'54,74”S
- 4953'39,32"O e elevação de 534 m, no estado de São Paulo (Figura 1). O morcego estava preso em um fio de arame farpado localizado a $2 \mathrm{~m}$ de altura em relação ao solo. Esta cerca era formada apenas por três fios e estava na parte superior de um alambrado de dois metros de altura, constituído por tela $\mathrm{n}^{\circ} 12$ com malha de duas polegadas, construída no entrono de uma granja desativada. $\mathrm{O}$ indivíduo estava preso pelo uropatágio na farpa do fio de arame mais próximo ao alambrado, há aproximadamente um dia, baseando-se no seu estado de decomposição pouco avançado. Foi possível observar que o animal tinha se enroscado na farpa durante o voo, porque o indivíduo girou $360^{\circ}$ ao redor do fio, causando perfurações em seu abdômen e pescoço na mesma farpa que prendeu o uropatágio. Possivelmente as lacerações em ambas as asas ocorrem após o animal se debater. Não foi encontrado nenhum agrupamento de morcegos abrigando-se nas proximidades da cerca onde obtive este registro.

FIGURA 1: Indivíduo da espécie M. molossus encontrado morto em uma cerca de arame farpado, na zona rural do município de São José do Rio Preto - SP, Brasil. Foto: Breviglieri C.P.B.

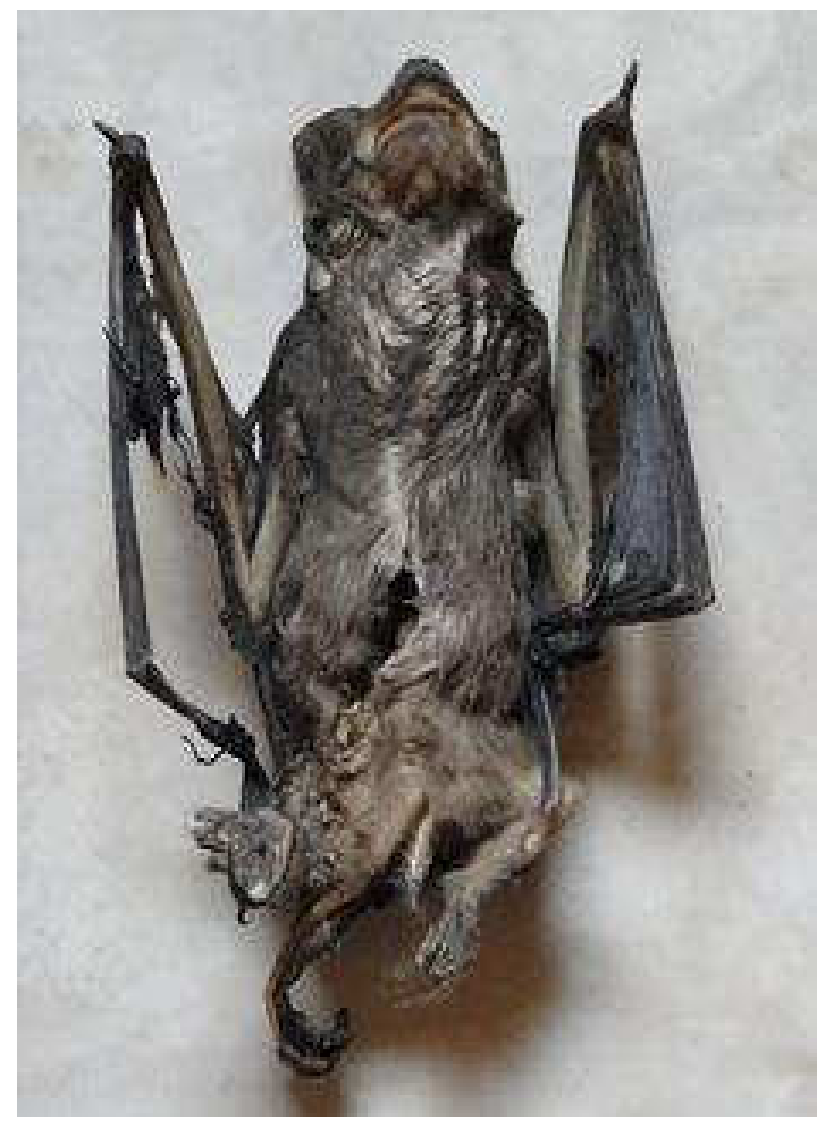


O segundo registro refere-se a um indivíduo macho da espécie Molossus rufus (E. Geoffroy, 1805), o qual apresentava 48,8 $\mathrm{mm}$ de comprimento do antebraço e pesava $30 \mathrm{~g}$. $\mathrm{O}$ animal foi encontrado morto no dia 15 de setembro de 2010, na zona rural de Fernandópolis sob a coordenada $20^{\circ} 20^{\prime} 12,84$ 'S - 50¹6’35,60”O, com elevação de $481 \mathrm{~m}$, na região noroeste do estado de São Paulo (Figura 2). Este indivíduo estava preso em um fio de arame farpado, a um metro de altura em relação ao solo, em uma cerca às margens de uma estrada não pavimentada, com paisagem predominantemente constituída por áreas abertas destinadas a criação de gado. O morcego estava enroscado apenas pelo uropatágio, e não apresentava outras escoriações em seu corpo. Este animal estava morto há aproximadamente uma semana, baseando-se em seu avançado estado de putrefação. É possível que este animal tenha morrido em função da desidratação devido à exposição prolongada ao sol.

FIGURA 2: Indivíduo da espécie $M$. rufus encontrado morto em uma cerca de arame farpado, na zona rural do município de Fernandópolis - SP, Brasil. Foto: Breviglieri C.P.B.

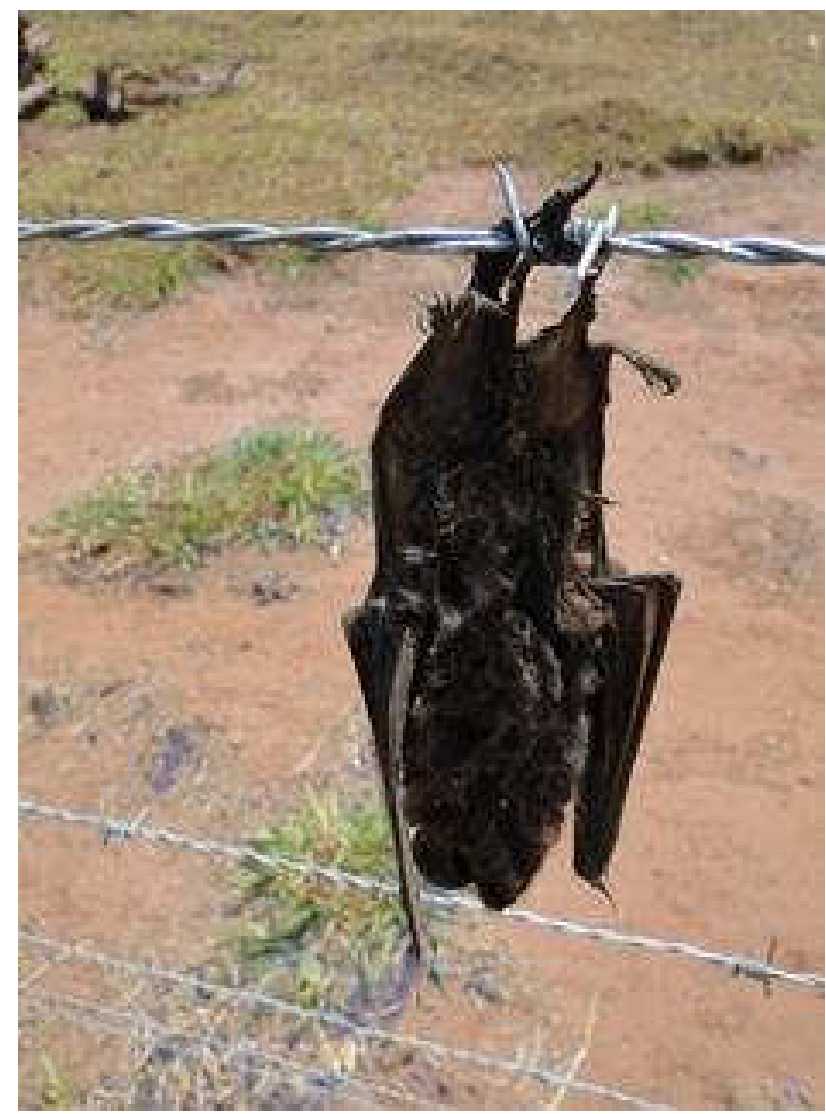

Foi constatada a presença de um agrupamento desta espécie em uma Paineira (Ceiba speciosa St.Hill.), de $10 \mathrm{~m}$ de altura, parcialmente morta, localizada a $20 \mathrm{~m}$ da cerca de arame farpado. $\mathrm{O}$ agrupamento era composto por três indivíduos, os quais foram observados no momento em que saiam do refúgio (por volta das 18h30min). Os animais passavam a menos de $2 \mathrm{~m}$ de distância da cerca no momento em que alçavam voo.

\section{Discussão}

Tanto M. molossus quanto $M$. rufus são considerados de hábito alimentar insetívoro aéreo (REIS et al., 2007), que forrageiam em locais abertos (VAUGHAN, 1966; FARNEY; FLEHARTY, 1969). Estas espécies podem abrigar-se em forros de casas, ocos de árvores, fendas em rochas, dentre outras estruturas construídas pelo homem (NOWAK, 1995; REIS et al., 2007). O voo muito alto e rápido característico da maioria das espécies de Molossidae (GREGORIN; TADDEI, 2002), juntamente com seu sistema de ecolocalização bem desenvolvido, reflete na pouca representação desta família em coleções científicas (GREGORIN; TADDEI, 2002), principalmente pela dificuldade de captura de exemplares através da metodologia de redes de neblina (KALKO et al., 1996; VOSS; EMMONS, 1996; GREGORIN; TADDEI, 2002; FABIAN; GREGORIN, 2007).

Entretanto, quando molossídeos emergem dos refúgios, apresentam baixa mobilidade de voo (BEST et al., 1996), facilitando sua captura por meio de redes neblina instaladas logo a frente dos acessos aos abrigos (BREVIGLIERI; PEDRO, 2010). Este comportamento poderia explicar o registro de $M$. rufus, morto após se enroscar na cerca de arame farpado adjacente ao abrigo. Outra hipótese que poderia explicar estes incidentes está relacionada com o hábito de forrageio destas espécies. Molossus molossus e M. rufus apresentam voo rápido (GREGORIN; TADDEI, 2002), mudando a direção rapidamente quando detectam uma possível presa (comunicação pessoal), portanto, estes animais podem colidir acidentalmente com obstáculos durante suas manobras, o que explicaria a presença destes morcegos enroscados em arames farpados (ver. NORQUAY et al., 2010). 
No caso de $M$. molossus, a presença de fontes de iluminação artificial localizada a quatro metros da cerca de arame farpado, pode ter contribuído para este evento. Fontes luminosas podem influenciar positivamente na atividade de morcegos insetívoros, devido à aglomeração de insetos atraídos pela luz (RYDELL, 2006), portanto, uma manobra brusca poderia ocasionar uma colisão com a cerca de arame farpado. Esta hipótese pode ser relacionada a diversos relatos de morcegos insetívoros encontrados mortos, enroscados em emaranhados de espinhos, ou aderidos em sementes de bardana (Arctium spp.) e/ou cactos, enquanto atacavam insetos que visitavam as flores destas plantas (FENTON, 1992; NORQUAY et al., 2010).

Diante destas informações, é possível propor que para evitar estes acidentes é fundamental a substituição das cercas de arame farpado por fio liso, principalmente em cercas que margeiam fragmentos florestais, árvores isoladas, possíveis abrigos ou fontes de alimentos utilizados por morcegos ou por outros animais. Esta medida deve ser considerada devido ao grande número de relatos, ao redor do mundo, descrevendo acidentes desta natureza. Se considerarmos ainda que muitos dos acidentes não são relatados, podemos estar subestimando seu efeito nas populações destes animais.

\section{Agradecimentos}

Agradeço aos revisores anônimos por seus valiosos comentários sobre o manuscrito.

\section{Referências}

ALLEN, G. T.; RAMIREZ, P. A review of bird deaths on barbed wire. The Wilson Bulletin, Michigan, n. 102, p. 553-558, 1990.

ANDERSON, H. L. Barbed wire impales another Great Homed Owl. Raptor Research, Albuquerque, v. 11, p. 71-72, 1977.

BEST, T. L.; KISER, W. M.; FREEMAN, P. W. Eumops perotis. Mammalian Species, Northampton, n. 534, p. 1-8, 1996.

BREVIGLIERI, C. P. B.; PEDRO, W. A. Diversidade de morcegos em três áreas do noroeste paulista, Brasil. Chiroptera Neotropical, Brasília, v. 16, p. 90-92, 2010.

DEDON, M.; BYRNE, S.; AYCRIGG, J.; HARTMAN, P. Bird mortality in relation to the Mare Island 115-kv transmission line: progress report 1988/1989. San Bruno: Department of the Navy, Western Division, Naval Facilities Engineering Command, Office of Environmental Management, 1989. 150 p.
DENYS, G. A. Hoary bat impaled on barbed wire. Jack-Pine Warbler, Michigan, v. 50, p. 63, 1972.

DISCHER, D. S.; BERNARDE, P. S.; FACURE, K. G. Mammalia, Chiroptera, Phyllostomidae, Vampyrum spectrum (Linnaeus, 1758): First record for the state of Rondônia, Brazil, and new prey records. Check List, Campinas, v. 5, n. 3, p. 394-395, 2009.

EMERSON, W. O. Destruction of birds by wires. Condor, Albuquerque, v. 6, p. 37-38, 1904.

FABIAN, M. E.; GREGORIN, R. Família Molossidae. In: REIS, N. R.; PERACCHI, A. L.; PEDRO, W. A.; LIMA, I. P. (Ed.). Morcegos do Brasil. 1. ed. Londrina: Universidade Estadual de Londrina, 2007. p. 149-166.

FARNEY, J.; FLEHARTY, E. D. Aspect ratio, loading, wing span, and membrane areas of bats. Journal of Mammalogy, Lawrence, v. 50, p. $362-367,1969$.

FENTON, M. B. Bats. New York: Checkmark Books, 1992. 208 p. GREGORIN, R.; TADDEI, V. A. Chave artificial para determinação de molossídeos brasileiros (Mammalia: Chiroptera). Mastozoologia Neotropical, Buenos Aires, v. 9, n. 1, p. 13-32, 2002.

KALKO, E. K. V.; HANDLEY, C. O.; HANDLEY, D. Organization, diversity, and long term dynamics of a Neotropical bat community. In: CODY, M. L.; SMALLWOOD, J. A. (Ed.). Long-term studies of vertebrate communities. San Diego: Academic Press, 1996. p. 503-553.

MACLEAN, J. J. Reducing the impact of barbed wire on wildlife. The Australasian Bat Society Newsletter, Sydney, v. 27, p. 33-36, 2006.

MCNICHOLL, M. K. Bats and birds stuck on burdock. The Prairie Naturalist, Brookings, n. 20, p. 157-160, 1988.

NAVO, K.; LAMANTIA-OLSON, N.; WOSTL, E.; GROVER, C.; HAYES, M.; DAY, R.; CLAWGES, R.; CARIS, C.; ESTEP, A. Multiple bats entangled on cactus. The Prairie Naturalist, Brookings, v.1, n. 43, p. 64-65, 2011

NORQUAY, K. J. O.; MENZIES, A. K.; MCKIBBIN, C. S.; TIMONIN, M. E.; BALOUN, D. E.; WILLIS, C. K. R. Silverhaired bats (Lasionycteris noctivagans) found ensnared on burdock (Arctium minus). Northwestern Naturalist, Washington, n. 91, p. 339-342, 2010.

RYDELL, J. Bats and their insect prey at streetlights. In: RICH, C.; LONGCORE, T. (Ed.). Ecological consequences of artificial night lighting. Washington: Island Press, 2006. p. 43-60.

VAN DER REE, R. Barbed wire fencing as a hazard for wildlife. The Victorian Naturalist, Melbourne, v. 116, p. 210-217, 1999.

VAUGHAN, T. A. Morphology and flight characteristics of molossid bats. Journal of Mammalogy, Lawrence, v. 47, p. 249260, 1966

VOSS, R. S.; EMMONS, L. H. Mammalian diversity in neotropical lowland rainforests: a preliminary assessment. Bulletin of American Museum Natural History, New York, v. 230, p. 1-250, 1996.

WISELY, A. N. Bat dies on barbed wire fence. Blue Jay, Saskatchewan, v. 36, p. 53, 1978. 